Open Access

\title{
Hypoxia induces calpain activity and degrades SMAD2 to attenuate TGF $\beta$ signaling in macrophages
}

\author{
Wei Cui ${ }^{1,2}$, Jie Zhou', Nathalie Dehne ${ }^{2}$ and Bernhard Brüne ${ }^{2^{*}}$
}

\begin{abstract}
Background: Under inflammatory conditions or during tumor progression macrophages acquire distinct phenotypes, with factors of the microenvironment such as hypoxia and transforming growth factor $\beta$ (TGF $\beta$ ) shaping their functional plasticity. TGF $\beta$ is among the factors causing alternative macrophage activation, which contributes to tissue regeneration and thus, resolution of inflammation but may also provoke tumor progression. However, the signal crosstalk between TGF $\beta$ and hypoxia is ill defined.

Results: Exposing human primary macrophages to TGF $\beta$ elicited a rapid SMAD2/SMAD3 phosphorylation. This early TGF $\beta$-signaling remained unaffected by hypoxia. However, with prolonged exposure periods to TGF $\beta /$ hypoxia the expression of SMAD2 declined because of decreased protein stability. In parallel, hypoxia increased mRNA and protein amount of the calpain regulatory subunit, with the further notion that TGF $\beta /$ hypoxia elicited calpain activation. The dual specific proteasome/calpain inhibitor MG132 and the specific calpain inhibitor 1 rescued SMAD2 degradation, substantiating the ability of calpain to degrade SMAD2. Decreased SMAD2 expression reduced TGF $\beta$ transcriptional activity of its target genes thrombospondin 1, dystonin, and matrix metalloproteinase 2.
\end{abstract}

Conclusions: Hypoxia interferes with TGF $\beta$ signaling in macrophages by calpain-mediated proteolysis of the central signaling component SMAD2.

Keywords: Hypoxia, TGFß, phospho-SMAD2, SMAD2 degradation, Calpain, Macrophages

\section{Introduction}

Macrophages are found throughout the body, where they contribute to tissue homeostasis and orchestrate innate as well as adaptive immune responses. They display a remarkable plasticity, which allows them to change their functional repertoire with regard to the environment they are facing. Numerous sub-phenotypes are described. Based on distinct functional roles and marker expression profiles an operative useful but over-simplifying nomenclature categorizes classical, i.e., pro-inflammatory and alternatively activated macrophages [1]. An alternatively activated macrophage phenotype is elicited by interleukin-4 (IL-4), IL-10, or transforming growth factor $ß$ (TGFß), which are produced by helper 2 or regulatory $\mathrm{T}$ cells, tumor cells, or macrophages themselves

\footnotetext{
* Correspondence: b.bruene@biochem.uni-frankfurt.de

${ }^{2}$ Institute of Biochemistry I, Faculty of Medicine, Goethe-University Frankfurt, 60590 Frankfurt, Germany

Full list of author information is available at the end of the article
}

during late stage immune responses and tumor cells. Monocyte-derived macrophages and tissue resident macrophages are found in tumors. These tumor-associated macrophages are known to support cancer progression rather than mounting anti-cancer responses. This tumorsupporting phenotype is elicited by cytokines like TGF $\beta$ [2].

TGFß controls proliferation, differentiation, and fosters fibrosis. TGFß binds to transforming growth factor receptor 2 (TGFBR2), which recruits and phosphorylates transforming growth factor receptor 1 (TGFBR1), thereby activating downstream signaling [3]. SMAD proteins mediate canonical TGFß signaling, while the PI3K-AKT, MAPK pathway conveys non-canonical signals [4]. The canonical pathway phosphorylates regulatory SMADs via receptor activation. In macrophages stimulation with TGF $\beta$ predominantly activates the regulatory SMAD2 and -3 but activation of SMAD1 and -5 was also observed [5]. Phosphorylated SMAD2 and SMAD3 share high affinity 
for SMAD4 and show oligomerization, which is necessary for nuclear translocation and activation of transcription [3]. SMAD7 is an inhibitory SMAD, which negatively controls this pathway.

TGFß plays a substantial role in tumor progression, acting both as a tumor suppressor and tumor promoter [6]. Early in tumorigenesis, TGFß arrests the cell-cycle, which is SMAD-dependent. With tumor progression, tumor cells often harbor inactivating mutations in the TGFß pathway, allowing them to overcome these growthinhibitory effects. In the tumor microenvironment, TGFß responsive cells secret cytokines and enzymes to promote EMT (epithelial mesenchymal transition), angiogenesis, tumor invasion, and metastasis. Besides, TGF $\beta$ also recruits and activates immune cells [7]. Of particular interest is the induction of an alternatively activated macrophage phenotype, which promotes tumor development $[8,9]$. TGFß-recruited macrophages are highly phagocytic for aberrant cells and thereby decrease the ability of tumor antigens to be delivered to the adaptive immune system [10]. Inhibition of NF-kB in TGFß-polarized macrophages accounts for their reduced ability to produce pro-inflammatory cytokines $[7,11]$.

At sites of inflammation, like in tumors, macrophages face hypoxia. Hypoxia promotes angiogenesis, fibrosis, and immune suppression [12]. Activation of the hypoxia-inducible factor (HIF) conveys many responses to hypoxia, by altering transcriptional outputs. HIF is a heterodimeric transcription factor composed of an ubiquitously expressed $\beta$-subunit and an oxygensensitive $\alpha$-subunit $[13,14]$. Three HIF- $\alpha$ subunits are identified in the human genome (HIF- $1 \alpha$, HIF- $2 \alpha$ or EPAS1, and HIF-3 $\alpha$ ), which all share the oxygendependent degradation domain (ODD). In welloxygenated tissue specific prolyl hydroxylases hydroxylate the ODD of the $\alpha$-subunit, allowing their recognition by the von Hippel-Lindau tumor suppressor and subsequent proteosomal degradation [13, 14]. Under hypoxic conditions HIF- $\alpha$ is stabilized, translocates to the nucleus and thus, promotes transcription of target genes to induce angiogenesis, ensures cell survival, restores oxygen homeostasis but also promotes fibrosis [15]. These functions overlap with TGFß-signaling, indicating some signal crosstalk $[6,12,16]$. In renal epithelial cells and prostate cancer cells TGFß provokes HIF- $1 \alpha$ accumulation, which in turn enhances expression of common target genes [17, 18]. In contrast, TGF $\beta$ alone failed to accumulate HIF- $1 \alpha$ in macrophages [19] even though the prolyl-hydroxylase-2 and -3 (PHD2/3) mRNA were decreased [20]. Although macrophages play an important role in fibrosis and immune suppression the impact of hypoxia on TGFß-signaling in macrophages is poorly understood. We provide evidence that TGFß-signaling under hypoxia is impaired, due to calpain-mediated SMAD2 degradation in macrophages.

\section{Results}

Hypoxia attenuates TGFß-induced SMAD2 activation in macrophages

Macrophages in the tumor microenvironment are exposed to hypoxia and TGFß but their signaling crosstalk has not been explored in detail. Therefore, we timedependently stimulated primary human macrophages with $10 \mathrm{ng} / \mathrm{mL}$ TGFß under normoxia vs. hypoxia and followed phosphorylation of SMAD2 (p-SMAD2) and SMAD3 (p-SMAD3) by Western blot analysis. Resting macrophage did not show SMAD2 phosphorylation (Fig. 1a, b), whereas TGFß provoked a rapid SMAD2 phosphorylation within 15 min. Phospho-SMAD2 reached its maximum after $1 \mathrm{~h}$ both under normoxia and hypoxia. After $4 \mathrm{~h}$, the level of p-SMAD2 gradually declined under hypoxia compared to normoxia. These differences became significant after $8 \mathrm{~h}$ (Fig. 1a, b). In contrast, the amount of p-SMAD3 rapidly increased upon TGFß addition, with phosphorylation not being affected by hypoxia. Although we noticed declining levels of p-SMAD3 at 4 to $8 \mathrm{~h}$, this occurred irrespective to the presence or absence of oxygen (Fig. 1c, d). Apparently, in TGF $\beta$-stimulated macrophages, only SMAD2, but not SMAD3, was sensitive to hypoxia. To explore this response for longer incubation times, we stimulated cells with TGF $\beta$ for 8 to 24 h at $20 \%$ or $1 \%$ $\mathrm{O}_{2}$. We observed decreased SMAD2 phosphorylation comparing 8 to 16 or $24 \mathrm{~h}$, an effect drastically enhanced by hypoxia (Fig. 1e, f). Using an antibody to detect total SMAD2 protein showed a progressively reduced expression under hypoxia from 8 to $24 \mathrm{~h}$ (Fig. 1e, g). Apparently, hypoxia reduced phosphorylation of SMAD2 at 8 to $24 \mathrm{~h}$ following TGF $\beta$-stimulation because of reduced SMAD2 protein expression.

\section{A decrease in SMAD2 under hypoxia is unrelated to TGFBR2 or SMAD7}

Several publications link SMAD activation to its subsequent degradation [21, 22]. Therefore, we started to validate TGFBR2 and SMAD7 as potential HIF target genes that were previously identified by ChIP-Seq and mRNA array experiments [23]. TGFBR2 is the receptor subunit that binds TGFß and phosphorylates the coreceptor TGFBR1 to initiate intracellular signaling. An altered expression level of TGFBR2 will alter the ratio of TGF $\beta$ to its receptor, thereby modulating downstream signaling. SMAD7 is an inhibitory SMAD, which competes with SMAD2/3 at the TGFß receptor to control TGFß signal transmission. Increased expression under hypoxia may reduce SMAD2 activation. First, we validated microarray data by quantitative PCR $8 \mathrm{~h}$ after TGFß and/or hypoxia stimulation and verified hypoxic 
A

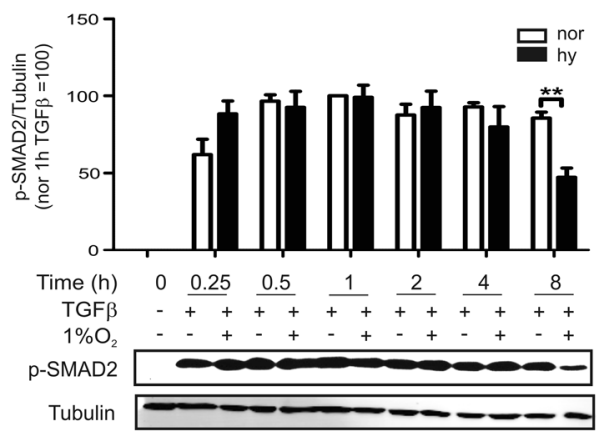

C

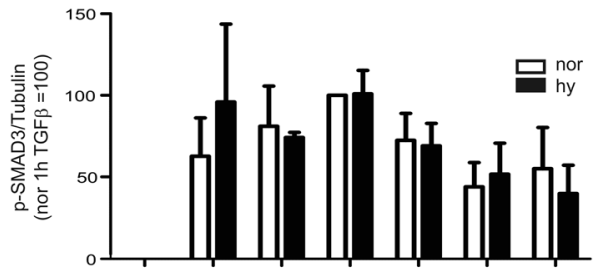

D
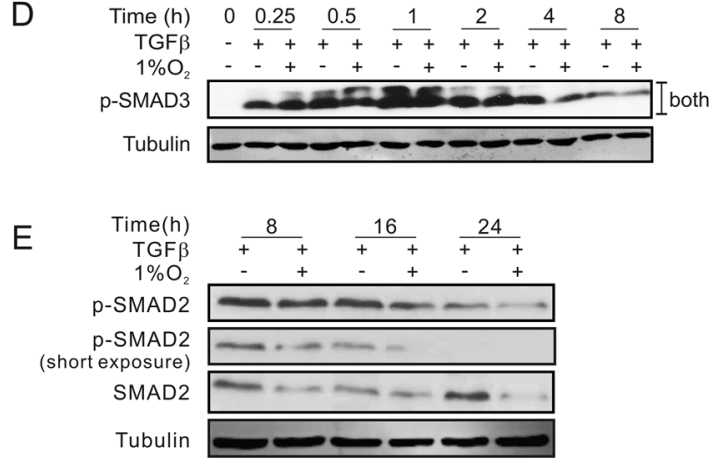

$\mathrm{F}$

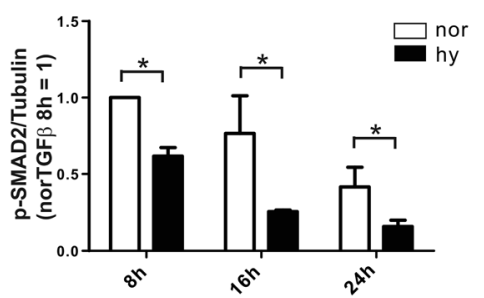

G

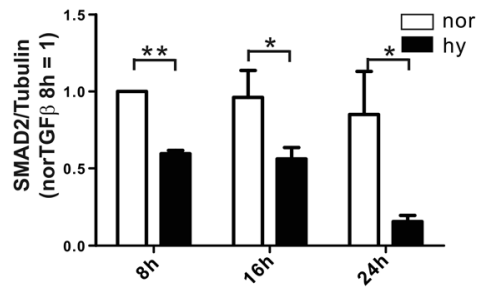

Fig. 1 Hypoxia attenuates TGFß-induced SMAD2 phosphorylation in macrophages. Time-dependent Western blot analysis $\mathbf{b}$, $\mathbf{d}$ and the corresponding statistical evaluation $\mathbf{a}, \mathbf{c}$ of SMAD2 $\mathbf{a}, \mathbf{b}$ and SMAD3 $\mathbf{c}$, $\mathbf{d}$ phosphorylation in human primary macrophages exposed to TGFß under normoxia (-; nor) or hypoxia (1 \% $\mathrm{O}_{2}$; hy). Macrophages were exposed to TGFß under normoxia vs. hypoxia for 8, 16, and 24 h, followed by (e) Western blot analysis of total SMAD2 and SMAD2 phosphorylation and corresponding statistical analysis $\mathbf{f}, \mathbf{g}$. Tubulin served as a loading control

induction of TGFBR2 mRNA (Fig. 2a). The knockdown of HIF- $1 \alpha$ or HIF- $2 \alpha$, which was verified by Western blot analysis (Additional file 1: Figure S1A, B), reduced hypoxic TGFBR2 mRNA induction. mRNA expression of TGFBR2 under hypoxia/TGFß was lower compared to the hypoxic response. In contrast, SMAD7 was predominantly induced by TGFß, with the signal not being altered by a HIF-knockdown (Fig. 2b). Hypoxia only showed a small mRNA induction of SMAD7, while induction by hypoxia/TGFß was comparable to TGFß alone. A knockdown of either HIF- $1 \alpha$ or HIF- $2 \alpha$ slightly reduced hypoxic induction of SMAD7 and significantly reduced induction by hypoxia/TGFß, suggesting the involvement of HIF in hypoxic SMAD7 mRNA regulation.

To determine the impact of hypoxia on TGFBR2 protein expression, we examined its surface appearance after $8 \mathrm{~h}$ by flow cytometry (Fig. 2c, d). TGFBR2 expression was neither altered by TGFß nor by the 


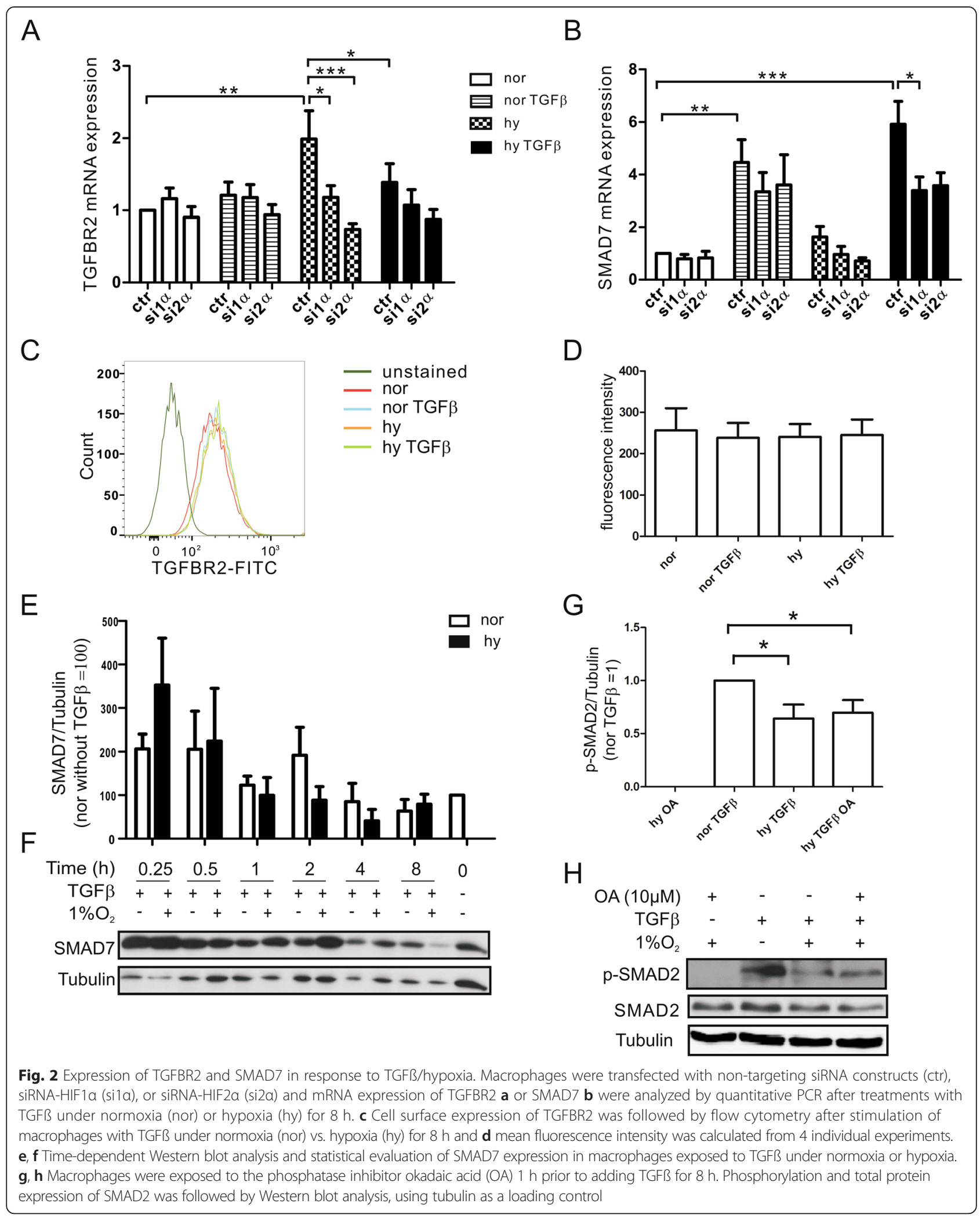


combination of hypoxia/TGFß, indicating that the SMAD2 decrease is unrelated to TGFBR2 expression. In addition, Western blot analysis verified that the expression of SMAD7 increased by TGFß at 15 to $30 \mathrm{~min}$, decreased after $1-2 \mathrm{~h}$ and remained low up to an $8 \mathrm{~h}$ incubation period. However, SMAD7 protein expression was similarly affected by TGFß supplied under normoxia and hypoxia (Fig. 2e, f). Thus, it appears that neither the expression of TGFBR2 nor of SMAD7 account for the specific effects of hypoxia seen at the level of SMAD2.

Next, we considered phosphatases to decrease SMAD2 phosphorylation. It was reported that PP2A dephosphorylates p-SMAD3 under hypoxia [24]. Supplying the PP2A inhibitor okadaic acid (OA) $1 \mathrm{~h}$ prior to addition of TGFß for $8 \mathrm{~h}$ failed to rescue the decrease of phoshorylated or total SAMD2 under TGFß/hypoxia (Fig. 2h). Excluding SMAD2 activation or dephoshorylation as an explanation for its decreased protein amount, we went on to analyze SMAD2 mRNA. In general, protein expression is either regulated by an altered mRNA expression, reduced translation, and/or enhanced degradation. SMAD2 mRNA expression was slightly induced but not reduced by hypoxia (Fig. 3a). Therefore, we examined protein stability by suppressing translation using $10 \mu \mathrm{g} /$ $\mathrm{ml}$ cycloheximide (CHX). SMAD2 was degraded faster, comparing hypoxia to normoxia (Fig. 3b). The half-life of SAMD2 under normoxia/TGFß was calculated to be $8.9 \pm 1.2 \mathrm{~h}$, while it was reduced to $5.1 \pm 0.6 \mathrm{~h}$ under hypoxia/TGFß (Fig. 3c). In conclusion, SMAD2 is destabilized under hypoxia in TGFß-stimulated macrophages.

\section{SMAD2 degradation under hypoxia is facilitated by calpain}

Considering increased degradation of SMAD2 under TGFß/hypoxia, we analyzed pathways being involved. Using bafilomycin A1 to inhibit lysosomal functions, SMAD2 degradation remained unaffected thus, ruling the involvement of the lysosomal compartment out (Fig. 4a, b). We then used MG132 to block proteasomaland calpain-dependent degradation systems. MG132 rescued SMAD2 degradation in response to TGFß/hypoxia, while lactacystin, a more specific proteasomal inhibitor, not affecting calpain, failed to restore SMAD2 expression under these conditions (Fig. 4c-f). Calpain is a calcium-dependent non-lysosomal cysteine proteolytic system that comprises a small regulatory subunit (CAPNS1, also known as calpain reg) and a large catalytic subunit ( $\mu$-calpain/m-calpain, also known as CAPN). We tested mRNA expression of CAPNS1 in macrophages stimulated with TGFß under normoxia and hypoxia. CAPNS1 mRNA was significantly upregulated by hypoxia or hypoxia/TGFß compared to normoxia or TGFß-stimulation (Fig. 4g). The mRNA increase became also apparent at the protein level (Fig. 4h). Expression of

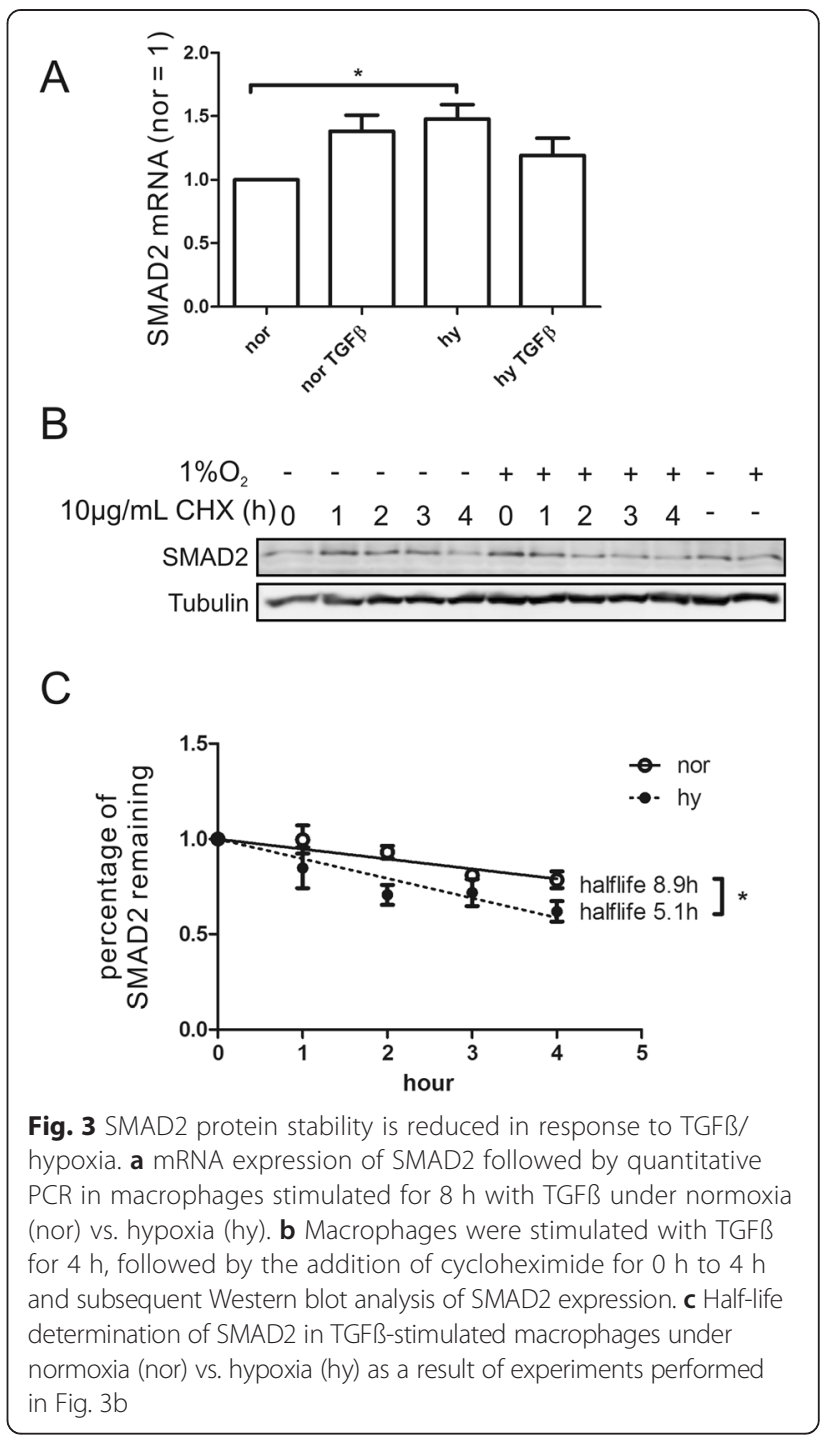

the catalytic subunit (CAPN1) was not affected by TGF $\beta$ or hypoxia nor their combination (Fig. 4i). Following macrophage activation with hypoxia/TGFß, holo-calpain $(80 \mathrm{KD})$ is proteolytically processed and the removal of 14 or 28 amino acids forms active calpain. Western blot analysis detected active calpain in macrophages stimulated with TGFß/hypoxia but not with TGF $\beta$ or hypoxia alone (Fig. 4j). Experiments with the specific calpain inhibitor 1 CPI $(10 \mu \mathrm{M})$, showed that decreased SMAD2 expression seen under hypoxia/TGFß was fully recovered when calpain activity was blocked (Fig. 4k). Conclusively, in TGFß-stimulated macrophages hypoxia promotes degradation of SMAD2 by increasing calpain activity.

To explore how hypoxia affects TGFß-induced transcriptional activity, we used a SMAD-specific reporter assay. Due to difficulties in transfecting primary human macrophages we used the J774A.1 mouse macrophage cell line, which responded with SMAD2 phosphorylation in 


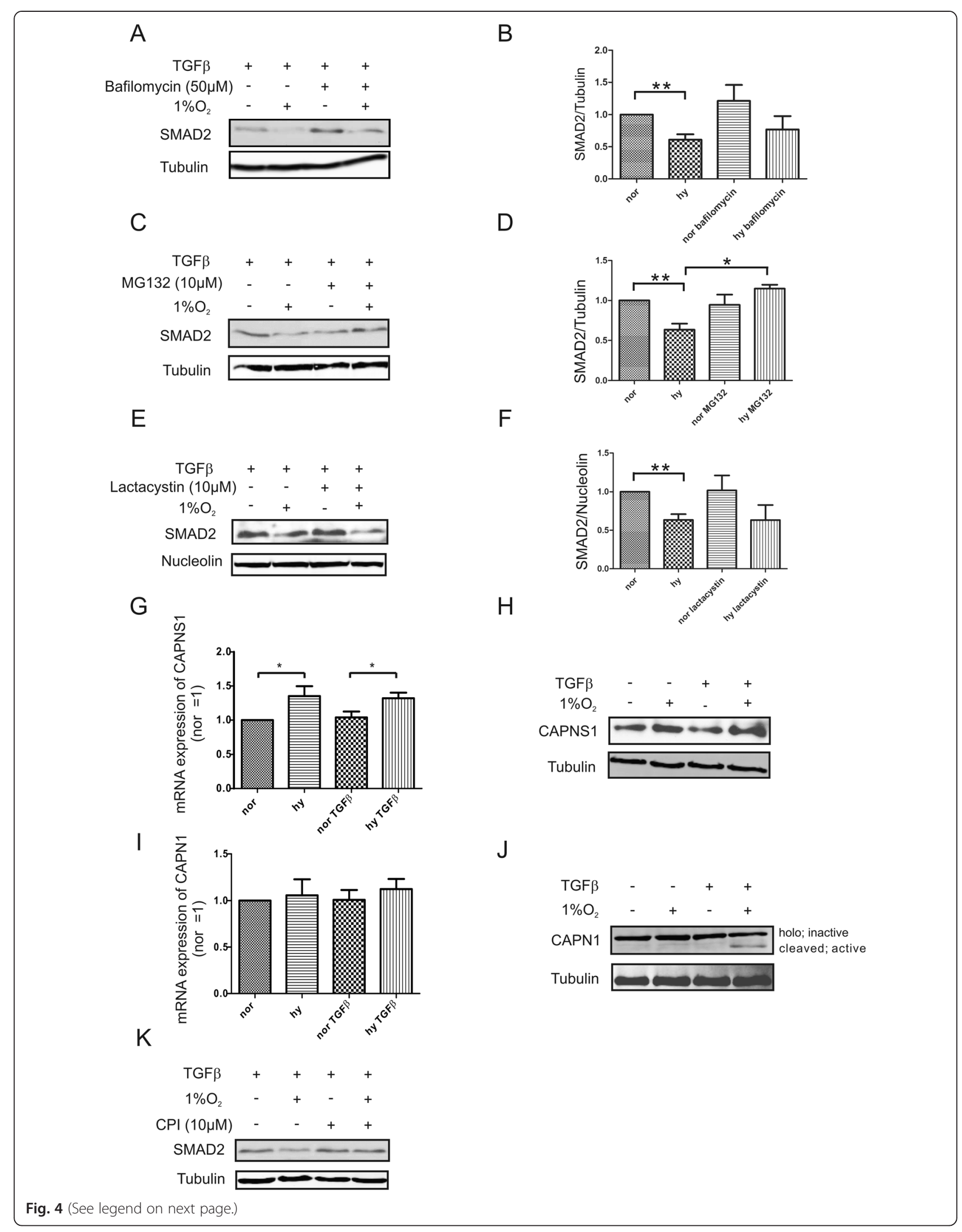


(See figure on previous page.)

Fig. 4 SMAD2 degradation under hypoxia is facilitated by calpain. a-f Macrophages were stimulated with TGFß under normoxia vs. hypoxia for $8 \mathrm{~h}$ in the presence of bafilomycin $\mathbf{a}, \mathbf{b}$, MG132 $\mathbf{c}$, d, or lactacystin e, f. $2 \mathrm{~h}$ after TGFß, inhibitors were added and incubations went on for $6 \mathrm{~h}$. Western blot analysis and statistical evaluation is presented. $\mathbf{g}$ mRNA expression of the calpain regulatory subunit (CAPNS1) was analyzed by quantitative PCR in macrophages exposed for $8 \mathrm{~h}$ to TGFß under normoxia (nor) vs. hypoxia (hy). $\mathbf{h}$ Western blot analysis of the calpain regulatory subunit after exposure to TGFß and/or hypoxia for $8 \mathrm{~h}$. i mRNA expression of the calpain 1 catalytic subunit (CAPN1) was analyzed by quantitative PCR in macrophages exposed for $8 \mathrm{~h}$ to TGFß under normoxia (nor) vs. hypoxia (hy). $\mathbf{j}$ Proteolytic cleavage of CAPN1 under TGFß/hypoxia was followed in macrophages by Western blot analysis after $8 \mathrm{~h}$. $\mathbf{k}$ Macrophages were exposed to the calpain inhibitor 1 (CPI) to follow SMAD2 degradation after stimulating cells for $8 \mathrm{~h}$ with TGFß under normoxia vs. hypoxia

response to TGFß, also showing a decrease in SMAD2 expression with TGFß/hypoxia under longer exposure times (Additional file 1: Figure S1C, D). Stimulation of J774A.1 cells, transfected with the SMAD reporter plasmid SEB4-Luc, with TGFß under hypoxia significantly reduced transcriptional activity compared to TGFß supplied under normoxia (Fig. 5a). We then analyzed expression of
SAMD2 and SMAD3 target genes in primary human macrophages. Thrombospondin 1 (TSP1), dystonin (DST), and matrix metalloproteinase 2 (MMP2) were reported to be selectively regulated by SMAD2 [25-27] and we confirmed their responsiveness to TGF $\beta$ (Fig. 5b, c, d). Induction was significantly reduced in macrophages stimulated with TGF $\beta$ at $1 \% \mathrm{O}_{2}$, reflecting the reduced expression of
A

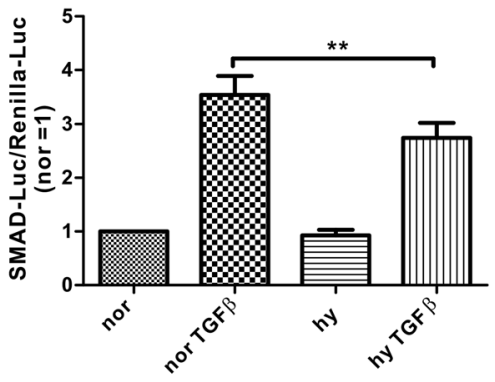

C

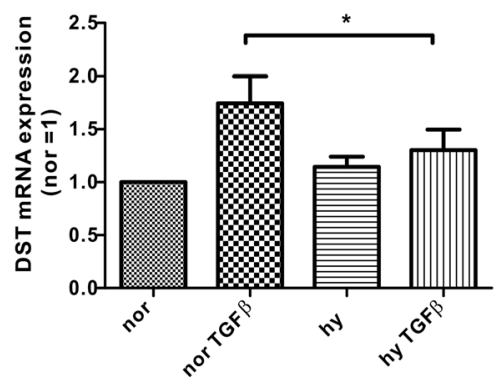

E

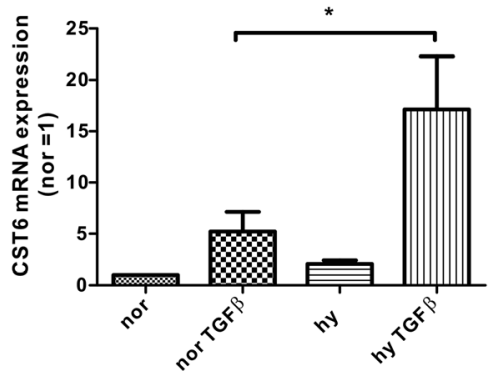

B

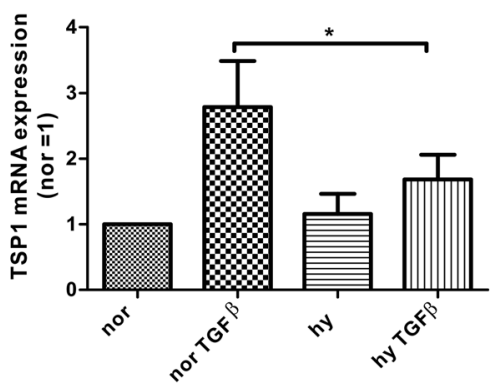

D

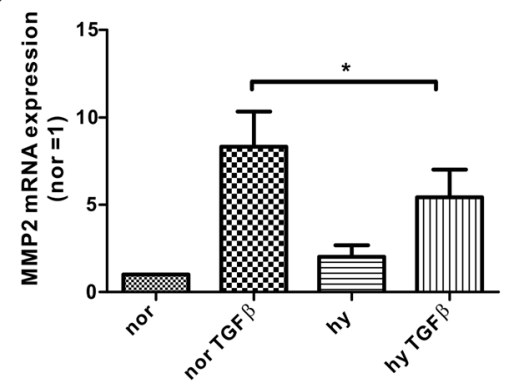

$\mathrm{F}$

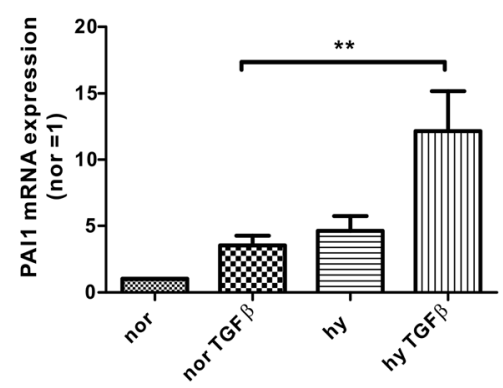

Fig. 5 Hypoxia reduces SMAD2-dependent transcription. a TGFß-induced transcriptional activity in J774A.1 cells transfected with a SBE4-Luc (4XSMAD binding element coupled to luciferase) as well as a Renilla-Luc plasmid. Stimulation was with TGFß under normoxia (nor) vs. hypoxia (hy) for 8 h. mRNA expression of $\mathbf{b}$ thrombospondin1 (TSP1), c dystonin DST, $\mathbf{d}$ matrix metalloproteinase (MMP2), e cystatin (CST6), and (f) plasminogen activator inhibitor 1 (PAl1) was analyzed by quantitative PCR in macrophages exposed for $8 \mathrm{~h}$ to TGFß under normoxia (nor) vs. hypoxia (hy) 
SMAD2. In contrast induction of SMAD3 target genes such as cystatin (CST6) and plasminogen activator inhibitor 1 (PAI1) [25] was not reduced under hypoxia (Fig. 5e, f). Apparently, only transcriptional responses of SMAD2 are selectively reduced by hypoxia in TGF $\beta$-stimulated human macrophages.

\section{Discussion}

In human macrophages, in contrast to epithelial and parenchymal cells $[17,18]$, periods of $8 \mathrm{~h}$ hypoxia or longer reduced TGFß-induced SMAD2 activation. Decreased phosphorylation of SMAD3, but not SMAD2, under hypoxia was noticed in HeLa cells and attributed to protein phosphatase 2A (PP2A) directly interacting with SMAD3 [24]. In macrophages the decrease of p-SMAD2 was not due to PP2A activation, altered TGFBR2 receptor activation, or inhibitory SMAD7 expression, but rather resulted from hypoxia-evoked destabilization of SMAD2. Regulation of SMAD2 degradation is complex. Smurf2 is an E3 ubiquitin ligase, directly interacting with activated SMAD2 and promoting its proteasomal degradation. Neural precursor cell expressed developmentally downregulated protein 4-2 (NEDD4-2), another E3 ubiquitin ligase like Smurf1 or 2, binds SMAD2 and SMAD3 but exclusively degrades SMAD2 [21]. In addition, TGF $\beta$ induced factor homeobox 1 (TGIF) interacting ubiquitin ligase 1 (Tiul1), induces SMAD2 to bind TGIF, which triggers SMAD2 degradation [22]. Our data add proteolysis by the calpain system also to account for alterations in the SMAD2 protein amount. Interestingly, Lo and coworkers studied the proteasomal degradation of SMAD2 [28]. Along our observations, their results suggested that lactacystin cannot rescue a SMAD2 decrease to the same extent as MG132, although they did not consider the calpain system.

The calpain system consists of a catalytic subunit ( $\mu$-calpain/m-calpain) and a common regulatory subnunit CAPNS1 as well as the inhibitory subunit calpastatin. After calcium binding, calpastatin detaches from the protein complex and calpain is activated by autolysis [29]. The calpain system plays important roles in macrophages. IFNY induces calpain mRNA and protein expression in U937 and THP1 cells [30]. Calpain cleaves iNOS and thus, affects inflammatory responses [31]. There are several reports that calpain activity is upregulated under hypoxia, with platelets being one typical example [32-35]. Hypoxia-induced thrombogenesis is associated with CAPNS1-dependent calpain activation in the platelet activation cascade [35]. In line, we noticed increased calpain activity under TGF $\beta$ /hypoxia by upregulating CAPNS1 in macrophages. The calpain regulatory subunit functions like a chaperone, assisting the catalytic subunit to fold properly [36]. We showed that combining TGFß and hypoxia is necessary to activate calpain in human macrophages as indicated in Fig. 4j, while hypoxia alone did not activate calpain. This is in line with findings showing that ischemia/hypoxia provokes an influx of $\mathrm{Ca}^{2+}$ into the cell $[32,35]$, but the $\mathrm{Ca}^{2+}$ increase is not sufficient to activate calpain $[29,37]$. It is well established that TGF $\beta$ could also increase the influx of $\mathrm{Ca}^{2+}$ into cells [38]. Presumably, a high expression of CAPNS1 and the influx of $\mathrm{Ca}^{2+}$ by the combined stimulation, hypoxia plus TGFß, promotes activation of calpain. Calpain digests proteins at a limited number of cleavage sites producing large polypeptide fragments rather than small peptides or amino acid [29]. Early studies suggested that preferred residues of the cleavage $\mathrm{P}_{2}$ position are Leu, Thr, and Val, and in the $P_{1}$ position are Lys, Thr, and Arg [39]. Following the $\mathrm{P}_{2}-\mathrm{P}_{1}$ rule, SMAD2 has 17 potential cleavage sites. In addition, calpain prefers small hydrophilic Ser residue at the $\mathrm{P}_{1}$ ' position. Considering this preference, there are still 4 possible cleavage sites at K46, Y101, R108, Y266 for calpain in SMAD2. On the other hand, there are indications that the specificity for calpain is not exclusively governed by amino acid but rather by the 3D conformation of the substrates $[29,39]$. Using currently available online tools at http://calpain.org for calpain substrates, amino acids at sites 166, 395, and 6 are the top 3 predicted cleavage sites with a score of $0.39,0.39,0.37$ respectively [40]. For the identification of the accurate cleavage sites more experiments are needed that would be beyond the scope of this work.

Our experimental conditions aimed to simulate conditions macrophages are facing when entering the tumor microenvironment, facing hypoxia and TGFß [41]. SMAD2 and SMAD3 have distinct transcriptional roles in the tumor [27, 42, 43]. SMAD2 provokes secretion of antiangiogenetic factors, such as thrombospondin 1 (TSP1, Fig. 5) as well as the VEGF-A antagonist sFlt-1, whereas SMAD3 induces the secretion of pro-angiogenetic factor, i.e., VEGF-A or plasminogen activator inhibitor 1 (PAI1, Fig. 5), promoting angiogenesis when SMAD2 is inactive $[26,44]$. In addition, TSP1 overexpression in tumor cells enhances the recruitment of pro-inflammatory macrophages and reduces tumor growth [45]. Conditional medium of SMAD2 knockdown fibroblast induces proliferation and in vivo deletion of SMAD2 in tumor cells generates a more aggressive phenotype compared to controls [26]. The decrease of SMAD2 under TGF $\beta$ /hypoxia in tumor-associated macrophages may add to their roles supporting angiogenesis and tumor progression.

Angiogenesis influences tumor growth but also enables metastasis [46]. Metastasis is a complex process requiring invasive growth of tumor cells, epithelial to mesenchymal transition (EMT), induction of angiogenesis, intra- and extravasation, and tumor growth at the secondary sites [47]. Matrix metalloproteases (MMPs) like MMP2 coordinate multiple steps of this process. In 
general they promote metastasis by allowing extracellular matrix remodeling and releasing growth factors like VEGF from the matrix. MMP2 was also implicated in endothelial cell apoptosis $[46,48]$. As full activation of MMP2 by the membrane type (MT)1-MMP provokes endothelial cell death and expression of MMP2 in endothelial cells and macrophages is reduced in acute hypoxia (Fig. 5d), one might speculate that this reduction prevents endothelial cell death under hypoxia when angiogenesis is usually enhanced [48, 49]. Other TGFß-induced genes involved in metastasis are enhanced under hypoxia since SMAD3 target genes like PAI-1 or CST6 (Fig. 5) are regulated by the hypoxiainducible factor (HIF) as well $[16,50]$. In a previous study we used ChIP-seq technology to identify HIF-1 and HIF-2 binding sites in primary human macrophages and identified, amongst others, CST6 and PAI-1 [23]. Apparently, in these cases TGFß/hypoxia enhances rather than decreases gene expression in macrophages. Thus, specifically attenuating SMAD2 under TGFß/hypoxia might have functional roles on macrophages biology and tumor progression.

\section{Conclusion}

Our results indicate that hypoxia attenuates SMAD2 activation in TGF $\beta$-stimulated macrophages (Fig. 6). Mechanistically, this results from degradation of SMAD2 by calpain.

\section{Material and methods}

Cell culture and incubation procedures

If not indicated otherwise, all chemicals were purchased from Sigma (Steinheim, Germany), while cell lines came from ATCC (LGC Promochem, Wesel, Germany). J774A.1 cells were cultured in Dulbecco's Modified Eagle Medium with $10 \%$ fetal calf serum, 100 $\mathrm{U} / \mathrm{ml}$ penicillin, and $100 \mu \mathrm{g} / \mathrm{ml}$ streptomycin (PAA Laboratories, Cölbe, Germany). Primary human monocytes were isolated from $50 \mathrm{ml}$ buffy coats (DRK Blutspendedienst Baden-Württemberg-Hessen, Frankfurt, Germany). Blood was layered on a Ficoll-Isopaque gradient $\left(\mathrm{P}=1077 \mathrm{~g} \mathrm{ml}^{-1}\right)$. The interphase containing peripheral blood mononuclear cells was obtained after centrifugation $(800 \times \mathrm{g}, 20 \mathrm{~min})$. Cells were recovered, washed twice in PBS, and left to adhere on culture dishes

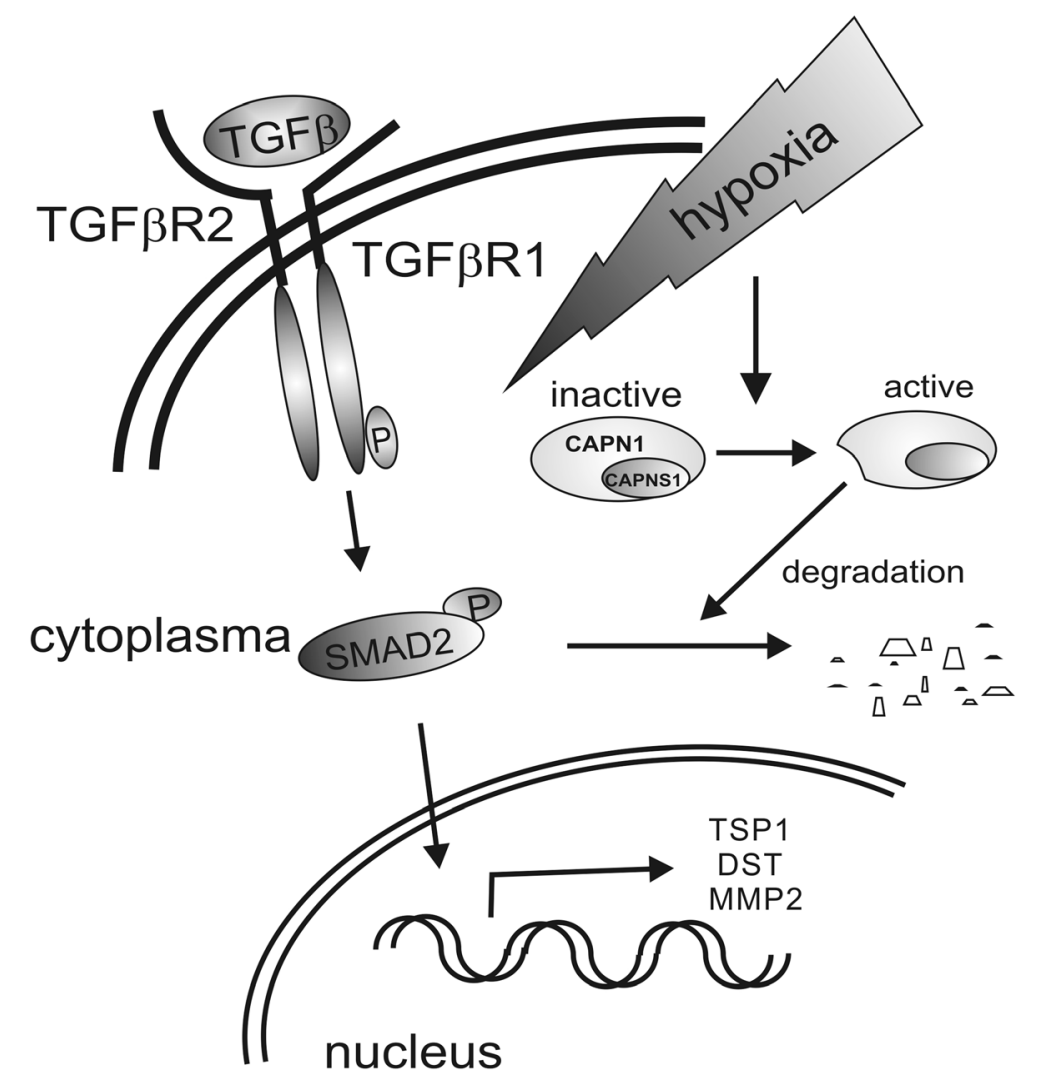

Fig. 6 Graphical abstract. TGF $\beta$ binds to its receptor (TGF $\beta R 1$ and TGF $\beta R 2$ ), induces phosphorylation of TGF $\beta$ R1 and downstream signaling molecules like SMAD2. Active SMAD2 translocates to the nucleus to provoke expression of target genes, i.e., TSP1, DST, and MMP2. Hypoxia in combination with TGF $\beta$ activates calpain to degrade SMAD2, which in turn attenuates SMAD2-dependent transcriptional activation and thus, diminishes the induction of TSP1, DST, and MMP2 
(Sarstedt, Nümbrecht, Germany) for $90 \mathrm{~min}$ at $37{ }^{\circ} \mathrm{C}$. Non-adherent cells were removed. The medium was changed to fresh RPMI 1640 medium, supplemented with $3 \%$ (v/v) human plasma (HP), $2 \mathrm{mM} \mathrm{L-glutamine,} 100 \mathrm{U} / \mathrm{ml}$ penicillin, and $100 \mu \mathrm{g} / \mathrm{ml}$ streptomycin (PAA Laboratories, Cölbe, Germany). Cells were kept at $37^{\circ} \mathrm{C}$ in a humidified atmosphere with $5 \% \mathrm{CO}_{2}$. Monocytes were differentiated with human plasma for 6-8 days before they were treated with $10 \mathrm{ng} / \mathrm{ml}$ TGFß. Purity of macrophage cultures was about $90 \%$. To establish hypoxic conditions cells were incubated in a hypoxic workstation with $1 \% \mathrm{O}_{2}, 94 \% \mathrm{~N}_{2}$, $5 \% \mathrm{CO}_{2}$ (Invivo2 400, Ruskinn Technology, Leeds U.K.). Protein stability was assessed by stimulating macrophage with TGFß for $4 \mathrm{~h}$, followed by $10 \mu \mathrm{g} / \mathrm{mL}$ cycloheximide (CHX). Samples were harvested after additional 1, 2, 3, or $4 \mathrm{~h}$. Degradation of SMAD2 was followed by the addition of $10 \mu \mathrm{M}$ lactacystin, $10 \mu \mathrm{M}$ MG132 (Z-Leu-Leu-Leu-al), or $50 \mu \mathrm{M}$ bafilomycin A1. After $2 \mathrm{~h}$ incubations with TGFß co-incubations with TGFß and inhibitors went on for $6 \mathrm{~h}$. Phosphatase PP2A was inhibited by $10 \mu \mathrm{M}$ okadaic acid (OA), preincubated for 1 h. $10 \mu \mathrm{M}$ calpain inhibitor 1 was added simultaneously together with TGFß to macrophages.

\section{Transfection of primary human macrophages}

For siRNA transfections human macrophages were seeded in 6-well plates as described above. $50 \mathrm{nM}$ siRNA against HIF- $1 \alpha$ (ON-TARGETplus SMART pool, Human HIF1A, Thermo Scientific, Karlsruhe, Germany), HIF-2 $\alpha$ (ON-TARGETplus SMART pool, Human EPAS1, Thermo Scientific), or non-targeting siRNA pool (GE Healthcare Dharmacon) was used with $16.8 \mu \mathrm{l}$ HiPerfect (Qiagen, Hilden, Germany) in $500 \mu \mathrm{l}$ medium with penicillin and streptomycin for each well. After adding the mix, cells were incubated for $24 \mathrm{~h}$. Then medium was removed and replaced by medium containing penicillin, streptomycin and human serum to start experiments.

\section{Western blot analysis}

Cells were lysed in lysis-buffer $(6.65 \mathrm{M}$ urea, $10 \%$ glycerol, $1 \%$ SDS, $10 \mathrm{mM}$ Tris/HCl $\mathrm{pH}$ 6.8, $\mathrm{pH}$ was adjusted to 7.4) and sonicated. After centrifugation $(16.000 \times \mathrm{g}, 10 \mathrm{~min})$ the protein content in the supernatants was determined by a protein assay kit (Bio-Rad, Munich, Germany), $70 \mu \mathrm{g}$ total protein was separated on $10 \%$ SDS gels and blotted on nitrocellulose membranes. The following antibodies were used: human pSMAD2 (\#3108, Cell Signaling Technology), human pSMAD3 (ab52903, Abcam), human SMAD7 (ab124890 Abcam), human SMAD2/3 (\#3102 Cell Signaling Technology), human calpain regulatory subunit (sc-32785, Santa Cruz), CAPN1 (C5736, Sigma), nucleolin (sc-13057, Santa Cruz), and B-tubulin (T4026, Sigma). Horseradish peroxidase-conjugated goat anti-rabbit or anti-mouse IgG
(Sigma) were used as secondary antibodies. Densitometry was performed using image J software (http://rsbweb.nih. gov/ij/). Briefly, rectangles were used to assess signal strength of each individual band and the background was measured by using the baseline tool and subtracted from individual values. Target protein expression was normalized to the loading control i.e., tubulin or nucleolin.

\section{Luciferase reporter activity}

The SBE4-Luc reporter plasmid was a gift from Bert Vogelstein (Addgene plasmid \# 16495) [51], while the renilla control vector pRL-SV40 was bought from Promega (Frankfurt, Germany). J774A.1 cells were transiently transfected with $0.2 \mu \mathrm{g}$ plasmid using JetPrime ${ }^{\mathrm{mm}}$ transfection reagent (Polyplus transfection, Illkirch, France) according to the manufacturer's protocol. After $16 \mathrm{~h}$, medium was changed and cells were starved for $2 \mathrm{~h}$ prior to stimulation. Afterwards, cells were lysed and firefly as well as renilla luciferase activities were determined using a Dual Luciferase kit assay (Promega) on a Mithras LB 940 luminometer (Berthold, Bad Wildbad, Germany).

\section{Flow cytometry (FACS)}

For TGFBR2 surface expression, single cell suspensions were generated from detached macrophages by digestion with accutase (PAA) for $10 \mathrm{~min}$ at $37^{\circ} \mathrm{C} .10^{5}$ cells were transferred to new tubes. Before staining, nonspecific antibody binding to $\mathrm{Fc} \gamma$ receptors was blocked with Fcy Block Receptor Binding Inhibitor (eBioscience, Frankfurt, Germany) for $15 \mathrm{~min}$ on ice, followed by staining with human TGFBR2 fluorescein-conjugated antibody (FAB241F,R\&D Systems, Germany) using standard protocols. We used an LSRFortessa Cell Analyzer (BD, US) and results were analyzed using FlowJo software 7.6.1 (Treestar, Ashland, OR, USA). To discriminate macrophages from other non-leukocyte cells, samples were stained with CD45 (BD Bioscience). For daily instrument calibration we used Cytometer Setup and Tracking beads (BD Biosciences).

\section{mRNA isolation and quantitative PCR}

Isolation of mRNA and quantitative PCR was performed as described in [52]. Primers are:

SMAD2, forward: CCGACACACCGAGATCCTAAC, reverse: GAGGTGGCGTTTCTGGAATATAA; SMAD7, forward: CCAGGCTCCAGAAGAAGTTG, reverse: CCAACTGCAGACTGTCCAGA; TGFBR2, forward: GGAAACTTGACTGCACCGTT, reverse: CTGCACATCGTCCTGTGG;

CAPNS1, forward:

GACACCCTGATCTGAAGACTGA, reverse: GCCTGCCACCTTTTGATGTT; 


\author{
CAPN1, forward: ACATGGAGGCCATCACTTTC \\ reverse: GGTCCACGTTGTTCCACTCT \\ TSP1, forward: CCAGATCAGGCAGACACAGA \\ reverse: AGTTGTCCCGTTCATTGAGG. \\ DST, forward: GATGCAGATCCGAAAACCCCT \\ reverse: CTCAGTGCGGTCCAGTTGTA \\ MMP2, forward: AGGGCACATCCTATGACAGC \\ reverse: ATTTGTTGCCCAGGAAAGTG \\ CST6, forward: CTACTTCCGAGACACGCACA \\ reverse: GGAACCACAAGGACCTCAAA \\ PAI1, forward: AGCTCCTTGTACAGATGCCG \\ reverse: ACAACAGGAGGAGAAACCCA \\ TBP, forward: GGGCCGCCGGCTGTTTAACT, \\ reverse: AGCCCTGAGCGTAAGGTGGCA.
}

\section{Statistics}

Experiments for mRNA expression and flow cytometry were performed in duplicate and repeated at least 3 times. Western blot analysis was performed at least 3 times. Data are expressed as means \pm SEM. Multigroup statistically significant differences were calculated after analysis of one-way variance (ANOVA) and Bonferroni's test. A statistically significant difference between two groups was calculated by paired $T$ test. Individual $P$ values are given in the figures $\left({ }^{*} P<0.05,{ }^{* *} P<0.01,{ }^{* * * *} P<0.001\right)$.

\section{Additional file}

Additional file 1: Figure S1. Western blot analysis of (A) HIF-1a and (B) HIF-2a of primary human macrophages transfected with non-targeted siRNA constructs (ctr), siRNA-HIF1a (si1a), or siRNA-HIF2a (si2a) exposed to normoxia vs. hypoxia $\left(1 \% \mathrm{O}_{2}\right)$ for $8 \mathrm{~h}$. (C) Western analysis of phospho SMAD2 in $\mathrm{J774}$ cells, exposed to TGFß under normoxia vs. hypoxia $(1 \%$ $\mathrm{O}_{2}$ ) for $8 \mathrm{~h}$. (D) Statistical analysis of data presented in Figure S1C.

\section{Competing interests}

The authors declare that they have no competing interests.

\section{Authors' contributions}

WC performed experiments; WC and ND designed experiments and analyzed data; WC, ND, BB wrote the manuscript; JZ revised the paper. All authors read and approved the final version of the manuscript. BB supervised the project.

\section{Authors' information}

Nathalie Dehne and Bernhard Brüne shared senior authorship.

\section{Acknowledgements}

This work was supported by the Deutsche Forschungsgemeinshaft (SFB 815, project A8), Deutsche Krebshilfe (111578), and Sander Foundation (2013.036.1)

Thanks to China Scholarship Council granting a stipend to Wei Cui.

\section{Author details}

${ }^{1}$ College of Life Sciences, Beijing Normal University, 100875 Beijing, China. ${ }^{2}$ Institute of Biochemistry I, Faculty of Medicine, Goethe-University Frankfurt, 60590 Frankfurt, Germany.
Received: 20 March 2015 Accepted: 12 June 2015

Published online: 04 July 2015

\section{References}

1. Murray PJ, Allen JE, Biswas SK, Fisher EA, Gilroy DW, Goerdt S, et al. Macrophage activation and polarization: nomenclature and experimental guidelines. Immunity. 2014;41(1):14-20.

2. Weigert A, Brüne B. Nitric oxide, apoptosis and macrophage polarization during tumor progression. Nitric Oxide. 2008;19(2):95-102.

3. Shi $Y$, Massagué J. Mechanisms of TGF- $\beta$ signaling from cell membrane to the nucleus. Cell. 2003;113(6):685-700.

4. Derynck R, Zhang YE. Smad-dependent and Smad-independent pathways in TGF- $\beta$ family signalling. Nature. 2003;425(6958):577-84.

5. Nurgazieva D, Mickley A, Moganti K, Ming W, Ovsyi I, Popova A, et al. TGF- $\beta 1$, but not bone morphogenetic proteins, activates Smad1/5 pathway in primany human macrophages and induces expression of proatherogenic genes. J Immunol. 2015;194(2):709-18.

6. Pickup M, Novitskiy S, Moses HL. The roles of TGF $\beta$ in the tumour microenvironment. Nat Rev Cancer. 2013;13(11):788-99.

7. Flavell RA, Sanjabi S, Wrzesinski SH, Licona-Limón P. The polarization of immune cells in the tumour environment by TGFB. Nat Rev Immunol. 2010;10(8):554-67.

8. Gong D, Shi W, Yi S-j, Chen H, Groffen J, Heisterkamp N. TGF $\beta$ signaling plays a critical role in promoting alternative macrophage activation. BMC Immunol. 2012;13(1):31.

9. Gigante M, Gesualdo L, Ranieri E. TGF-beta: a master switch in tumor immunity. Curr Pharm Des. 2012;18(27):4126-34

10. Byrne SN, Knox MC, Halliday GM. TGF $\beta$ is responsible for skin tumour infiltration by macrophages enabling the tumours to escape immune destruction. Immunol Cell Biol. 2007;86(1):92-7.

11. Mantovani A, Sica A. Macrophages, innate immunity and cancer: balance, tolerance, and diversity. Curr Opin Immunol. 2010;22(2):231-7.

12. Harris AL. Hypoxia - a key regulatory factor in tumour growth. Nat Rev Cancer. 2002;2(1):38-47.

13. Ratcliffe PJ. Oxygen sensing and hypoxia signalling pathways in animals: the implications of physiology for cancer. J Physiol. 2013;591(8):2027-42.

14. Brüne B, Dehne N, Grossmann N, Jung M, Namgaladze D, Schmid T, et al. Redox control of inflammation in macrophages. Antioxid Redox Signal. 2013;19(6):595-637.

15. Nath B, Szabo G. Hypoxia and hypoxia inducible factors: diverse roles in liver diseases. Hepatology. 2012;55(2):622-33.

16. Ueno M, Maeno T, Nomura M, Aoyagi-lkeda K, Matsui H, Hara K, et al. Hypoxia-inducible factor-1a mediates TGF- $\beta$-induced PAI-1 production in alveolar macrophages in pulmonary fibrosis. Am J Physiol Lung Cell Mol Physiol. 2011;300(5):L740-52.

17. Basu RK, Hubchak S, Hayashida T, Runyan CE, Schumacker PT, Schnaper HW. Interdependence of HIF-1 $\mathrm{a}$ and TGF- $\beta /$ Smad3 signaling in normoxic and hypoxic renal epithelial cell collagen expression. Am J Physiol Renal Physiol. 2011;300(4):F898-905.

18. Chae K, Kang M, Lee J, Ryu B, Lee M, Her N, et al. Opposite functions of HIF-a isoforms in VEGF induction by TGF- $\beta 1$ under non-hypoxic conditions. Oncogene. 2011;30(10):1213-28.

19. Herr B, Zhou J, Werno C, Menrad H, Namgaladze D, Weigert A, et al. The supernatant of apoptotic cells causes transcriptional activation of hypoxia-inducible factor-1a in macrophages via sphingosine-1-phosphate and transforming growth factor- $\beta$. Blood. 2009;114(10):2140-8.

20. Dehne N, Tausendschön M, Essler S, Geis T, Schmid T, Brüne B. IL-4 reduces the proangiogenic capacity of macrophages by down-regulating HIF-1a translation. J Leukoc Biol. 2014;95(1):129-37.

21. Kuratomi G, Komuro A, Goto K, Shinozaki M, Miyazawa K, Miyazono K, et al. NEDD4-2 (neural precursor cell expressed, developmentally down-regulated 4-2) negatively regulates TGF-beta (transforming growth factor-beta) signalling by inducing ubiquitin-mediated degradation of Smad2 and TGF-beta type I receptor. Biochem J. 2005;386:461-70.

22. Seo SR, Lallemand F, Ferrand N, Pessah M, L'Hoste S, Camonis J, et al. The novel E3 ubiquitin ligase Tiul1 associates with TGIF to target Smad2 for degradation. EMBO J. 2004:23(19):3780-92.

23. Tausendschön M, Rehli M, Dehne N, Schmidl C, Döring C, Hansmann M-L, et al. Genome-wide identification of hypoxia-inducible factor-1 and-2 binding sites in hypoxic human macrophages alternatively activated by IL-10. Biochim Biophys Acta. 2015;1849(1):10-22. 
24. Heikkinen PT, Nummela M, Leivonen SK, Westermarck J, Hill CS, Kahari VM, et al. Hypoxia-activated Smad3-specific Dephosphorylation by PP2A. J Biol Chem. 2009;285(6):3740-9.

25. Koinuma D, Tsutsumi S, Kamimura N, Taniguchi H, Miyazawa K, Sunamura $M$, et al. Chromatin immunoprecipitation on microarray analysis of Smad2/3 binding sites reveals roles of ETS1 and TFAP2A in transforming growth factor $\beta$ signaling. Mol Cell Biol. 2009;29(1):172-86.

26. Nakagawa T, Li JH, Garcia G, Mu W, Piek E, Böttinger EP, et al. TGF- $\beta$ induces proangiogenic and antiangiogenic factorsvia parallel but distinct Smad pathways1. Kidney Int. 2004;66(2):605-13.

27. Piek E, Ju WJ, Heyer J, Escalante-Alcalde D, Stewart CL, Weinstein M, et al. Functional characterization of transforming growth factor $\beta$ signaling in Smad2-and Smad3-deficient fibroblasts. J Biol Chem. 2001;276(23):19945-53.

28. Lo RS, Massagué J. Ubiquitin-dependent degradation of TGF- $\beta$ activated Smad2. Nat Cell Biol. 1999;1(8):472-8.

29. Goll DE, Thompson VF, Li H, Wei W, CONG J. The calpain system. Physiol Rev. 2003:83(3):731-801.

30. Deshpande RV, Goust J-M, Chakrabarti AK, Barbosa E, Hogan EL, Banik NL. Calpain expression in lymphoid cells increased mRNA and protein levels after cell activation. J Biol Chem. 1995;270(6):2497-505.

31. Walker G, Pfeilschifter J, Otten U, Kunz D. Proteolytic cleavage of inducible nitric oxide synthase (iNOS) by calpain I. Biochim Biophys Acta. 2001;1568(3):216-24.

32. Zheng $X$, Zhou A-X, Rouhi $P$, Uramoto H, Borén J, Cao Y, et al. Hypoxia-induced and calpain-dependent cleavage of filamin A regulates the hypoxic response. Proc Natl Acad Sci. 2014;111(7):2560-5.

33. Iwamoto H, Miura T, Okamura T, Shirakawa K, Iwatate M, Kawamura S, et al. Calpain inhibitor-1 reduces infarct size and DNA fragmentation of myocardium in ischemic/reperfused rat heart. J Cardiovasc Pharmacol. 1999;33(4):580-6.

34. Zhang J, Patel JM, Block ER. Hypoxia-specific upregulation of calpain activity and gene expression in pulmonary artery endothelial cells. Am J Physiol Lung Cell Mol Physiol. 1998;275(3):L461-8.

35. Tyagi T, Ahmad S, Gupta N, Sahu A, Ahmad Y, Nair V, et al. Altered expression of platelet proteins and calpain activity mediate hypoxia-induced prothrombotic phenotype. Blood. 2014;123(8):1250-60.

36. Zimmerman UJP, Boring L, Pak UH, Mukerjee N, Wang KK. The calpain small subunit gene is essential: its inactivation results in embryonic lethality. IUBMB Life. 2000;50(1):63-8.

37. Zhou J, Köhl R, Herr B, Frank R, Brüne B. Calpain mediates a von hippel-lindau protein-independent destruction of hypoxia-inducible factor-1a. Mol Biol Cell. 2006:17(4):1549-58.

38. Muldoon LL, Rodland K, Magun B. Transforming growth factor beta and epidermal growth factor alter calcium influx and phosphatidylinositol turnover in rat-1 fibroblasts. J Biol Chem. 1988;263(35):18834-41.

39. Tompa P, Buzder-Lantos P, Tantos A, Farkas A, Szilágyi A, Bánóczi Z, et al. On the sequential determinants of calpain cleavage. J Biol Chem. 2004;279(20):20775-85.

40. Ono $Y$, Sorimachi $H$, Mamitsuka $H$. Calpain cleavage prediction using multiple kernel learning. PLoS One. 2011;6(5):e19035.

41. Whiteside T. The tumor microenvironment and its role in promoting tumor growth. Oncogene. 2008;27(45):5904-12.

42. LaGamba D, Nawshad A, Hay ED. Microarray analysis of gene expression during epithelial-mesenchymal transformation. Dev Dyn. 2005:234(1):132-42

43. Kretschmer A, Moepert K, Dames S, Sternberger M, Kaufmann J, Klippel A. Differential regulation of TGF- $\beta$ signaling through $\mathrm{Smad} 2, \mathrm{Smad} 3$ and Smad4. Oncogene. 2003;22(43):6748-63.

44. Petersen M, Pardali E, Van Der Horst G, Cheung H, Van Den Hoogen C, Van Der Pluijm G, et al. Smad2 and Smad3 have opposing roles in breast cancer bone metastasis by differentially affecting tumor angiogenesis. Oncogene. 2009:29(9):1351-61.

45. Martin-Manso G, Galli S, Ridnour LA, Tsokos M, Wink DA, Roberts DD. Thrombospondin 1 promotes tumor macrophage recruitment and enhances tumor cell cytotoxicity of differentiated U937 cells. Cancer Res. 2008;68(17):7090-9.

46. Deryugina El, Quigley JP. Tumor angiogenesis: MMP-mediated induction of intravasation-and metastasis-sustaining neovasculature. Matrix Biol. 2015 doi:10.1016/j.matbio.2015.04.004.
47. Jiang W, Sanders A, Katoh M, Ungefroren H, Gieseler F, Prince M, et al. Tissue invasion and metastasis: molecular, biological and clinical perspectives. Semin Cancer Biol. 2015. doi: 10.1016/j.semcancer.2015.03.008.

48. Shapiro S, Khodalev O, Bitterman H, Auslender R, Lahat N. Different activation forms of MMP-2 oppositely affect the fate of endothelial cells. Am J Physiol Cell Physiol. 2010;298(4):C942-51.

49. Ben-Yosef $Y$, Lahat N, Shapiro S, Bitterman H, Miller A. Regulation of endothelial matrix metalloproteinase-2 by hypoxia/reoxygenation. Circ Res. 2002;90(7):784-91.

50. Chowdhury UR, Samant RS, Fodstad O, Shevde LA. Emerging role of nuclear protein 1 (NUPR1) in cancer biology. Cancer Metastasis Rev. 2009;28(1-2):225-32.

51. Zawel L, Le Dai J, Buckhaults P, Zhou S, Kinzler KW, Vogelstein B, et al. Human Smad3 and Smad4 are sequence-specific transcription activators. Mol Cell. 1998;1(4):611-7.

52. Jennewein C, Kuhn A-M, Schmidt MV, Meilladec-Jullig V, von Knethen A, Gonzalez FJ, et al. Sumoylation of peroxisome proliferator-activated receptor $\gamma$ by apoptotic cells prevents lipopolysaccharide-induced NCoR removal from $\mathrm{KB}$ binding sites mediating transrepression of proinflammatory cytokines. J Immunol. 2008;181(8):5646-52.

\section{Submit your next manuscript to BioMed Central and take full advantage of:}

- Convenient online submission

- Thorough peer review

- No space constraints or color figure charges

- Immediate publication on acceptance

- Inclusion in PubMed, CAS, Scopus and Google Scholar

- Research which is freely available for redistribution 